\title{
The Effect of General Relativity on Hyperbolic Orbits and Its Application to the Flyby Anomaly
}

\author{
Lorenzo Iorio \\ Istituto Nazionale di Fisica Nucleare (INFN), Sezione di Pisa, Viale Unità di Italia 68, 70125 Bari, Italy \\ Correspondence should be addressed to Lorenzo Iorio, lorenzo.iorio@libero.it
}

Received 24 November 2008; Accepted 17 January 2009

We investigate the impact of the general relativistic gravitoelectromagnetic forces on hyperbolic orbits around a massive spinning body. The gravitomagnetic field, causing the well-known Lense-Thirring precessions of elliptic orbits, is generated by the spin S of the central body. It deflects and displaces the trajectories differently according to the mutual orientation of $\mathbf{S}$ and the orbital angular momentum $\mathbf{L}$ of the test particle. The gravitoelectric force, which induces the Einstein precession of the perihelion of the orbit of Mercury, always deflects the trajectories inward irrespective of the $\mathbf{L}-\mathbf{S}$ orientation. We numerically compute their effect on the range $r$, radial and transverse components $v_{r}$ and $v_{\tau}$ of the velocity, and speed $v$ of the NEAR spacecraft at its closest approach with the Earth in January 1998 when it experienced an anomalous increase of its asymptotic outgoing velocity $v_{\infty}$ of $13.46 \pm 0.01 \mathrm{~mm} \mathrm{sec}^{-1}$; while the gravitoelectric force was modeled in the software used to process the NEAR data, this was not done for the gravitomagnetic one. The range rate and the speed are affected by general relativistic gravitoelectromagnetism at $10^{-2}$ (gravitoelectric) to $10^{-5}$ (gravitomagnetic) $\mathrm{mm} \mathrm{sec}^{-1}$ levels. The changes in the range are of the order of $10^{-2}$ (gravitomagnetic) to $10^{1}$ (gravitoelectric) $\mathrm{mm}$.

Copyright (C) 2009 Lorenzo Iorio. This is an open access article distributed under the Creative Commons Attribution License, which permits unrestricted use, distribution, and reproduction in any medium, provided the original work is properly cited.

\section{Introduction}

We will investigate the effects of general relativity, in its weak-field and slow-motion approximation, on unbound, hyperbolic orbits of test particles approaching a body of mass $M$ and angular momentum $\mathbf{S}$. We will consider both the gravitomagnetic and the gravitoelectric relativistic forces induced by the stationary and static components, respectively, of the field of $M$ [1].

The results obtained will be applied to some realistic planet-spacecraft scenarios in the Solar System to see if the predicted effects fall within the current or future sensitivity level of the ranging techniques. In particular, we will examine the so-called flyby anomaly consisting of unexplained changes of the asymptotic outgoing velocities of some spacecraft (Galileo, NEAR, Cassini, and MESSENGER) that occurred at their closest approaches with the Earth $[2,3]$.

Let us recall some basics of the Newtonian hyperbolic orbit [4] which represents, in this case, the reference unperturbed path; see Figure 1. Its equation is

$$
r=\frac{p}{1+e \cos f}
$$

where $p=a\left(e^{2}-1\right)$ is the semilatus rectum, and $f$ is the true anomaly considered positive in the anticlockwise direction from the point of closest approach $A ; a$ is the semimajor axis and $e>1$. The speed is

$$
v=\sqrt{G M\left(\frac{2}{r}+\frac{1}{a}\right)},
$$

so that

$$
v_{\infty}=\sqrt{\frac{G M}{a}} .
$$

The asymptotic ingoing and outgoing speeds, which are equal to $v_{\infty}$ in the unperturbed case, are denoted with $v_{\infty \mathrm{i}}$ and $v_{\infty 0}$, respectively. The angle $\phi$ between the $\mathbf{r}$ and $\mathbf{v}$ is defined by

$$
\begin{aligned}
\sin \phi & =\frac{1+e \cos f}{\sqrt{1+e^{2}+2 e \cos f}}, \\
\cos \phi & =-\frac{e \sin f}{\sqrt{1+e^{2}+2 e \cos f}} .
\end{aligned}
$$




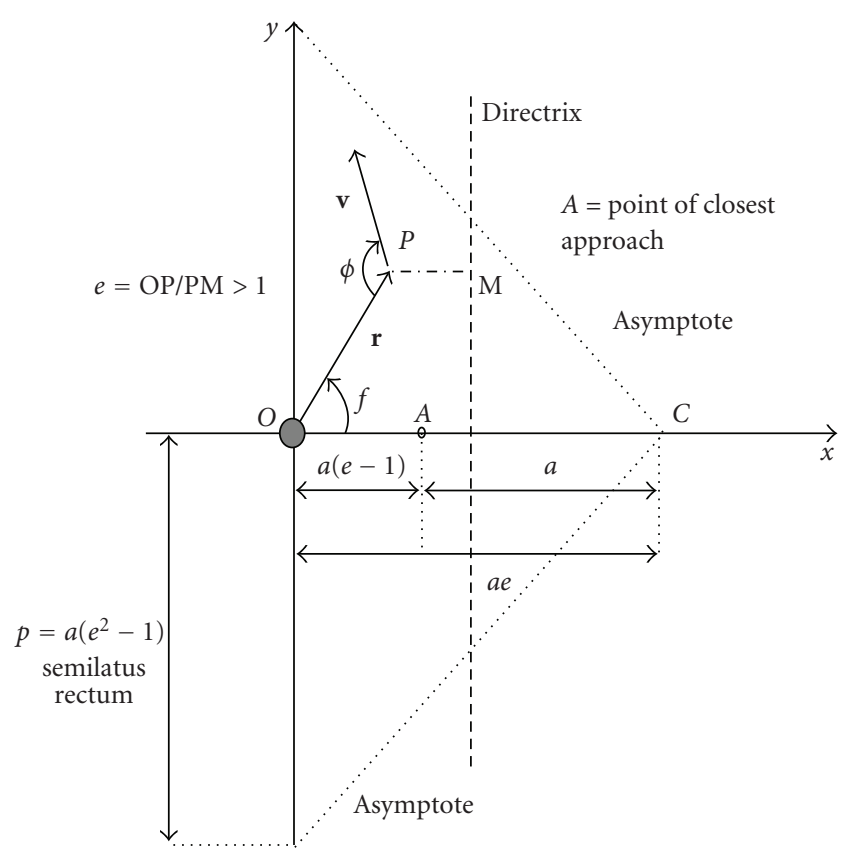

FIGURE 1: Essential features of the unperturbed Newtonian hyperbola. $P$ is the position of a test particle at time $t$ with respect to the body located at the origin of the chosen reference frame. The smallest distance is $r_{\min }=a(e-1)$. The angle between $\mathbf{r}$ and $\mathbf{v}$ is $\phi$. $\mathrm{M}$ here is not to be confused with the central body of mass $M$.

For $x=0, y= \pm p$, that is, $f=\bar{f}= \pm \pi / 2$, it is

$$
\begin{aligned}
& v_{x 0}=\bar{v} \sin \bar{\phi}=\sqrt{\frac{G M}{p}}, \\
& v_{y 0}=\bar{v} \cos \bar{\phi}=\mp e \sqrt{\frac{G M}{p}} .
\end{aligned}
$$

\section{The Gravitomagnetic Force}

In this section, we will deal with the effect of the general relativistic gravitomagnetic force on the hyperbolic motion of a test particle approaching a spinning body of mass $M$ and angular momentum $\mathbf{S}$.

Let us briefly recall that, in the weak-field and slowmotion linear approximation of general relativity, the offdiagonal components $g_{0 i}, i=1,2,3$ of the space-time metric tensor, related to the mass-energy currents of the source, induce a gravitomagnetic field $\mathbf{B}_{\mathrm{g}}[1]$ by analogy with the magnetic field caused by moving electric charges in the linear Maxwellian electromagnetism. Far from an isolated rotating body, the gravitomagnetic field becomes [5]

$$
\mathbf{B}_{\mathrm{g}}=-\frac{G}{c r^{3}}[\mathbf{S}-3(\mathbf{S} \cdot \hat{\mathbf{r}}) \hat{\mathbf{r}}],
$$

where $G$ is the Newtonian gravitational constant and $c$ is the speed of light in vacuum. It exerts the noncentral Lorentzlike acceleration [1]

$$
\mathbf{A}^{\mathrm{GM}}=-\frac{2}{c} \mathbf{v} \times \mathbf{B}_{\mathrm{g}}
$$

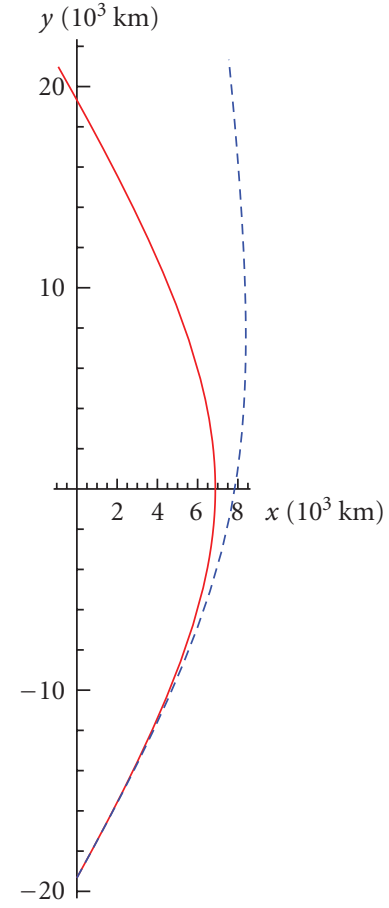

Figure 2: Effect of the gravitomagnetic force on the hyperbolic motion of a test particle around an astronomical rotating body located at the origin of the depicted frame. The body spin $\mathbf{S}$ is directed along the positive $z$ axis, that is, outside the figure. Red line: unperturbed hyperbola. Blue dashed line: perturbed orbit. For illustrative purposes, we choose the Earth as central body and rescaled the magnitude of its gravitomagnetic force by $10^{10}$ so that $A^{\mathrm{GM}} / A^{\mathrm{N}}=0.4$ at perigee. We adopted the initial conditions $x_{0}=$ $0, y_{0}=-p=-a\left(e^{2}-1\right), z_{0}=0, v_{x 0}>0, v_{y 0}>0, v_{z 0}=0$; the particle moves in the equatorial plane of the spinning Earth in such a way that the radius vector rotates in the same sense with respect to the Earth, that is, anticlockwise. We used $a=8,493.326 \mathrm{~km}$, $e=1.81$. The perturbed orbit is deflected outward with respect to the unperturbed one.

upon a test particle moving with velocity $\mathbf{v}$. For ordinary astronomical bodies like, for example, the Earth and the Sun, $A^{\mathrm{GM}}$ is many orders of magnitude smaller than the Newtonian monopole $A^{\mathrm{N}}=G M / r^{2}$, so that it can be considered as a small perturbation.

The action of (7) in the case of unperturbed close orbits, giving rise to, for example, the Lense-Thirring precession of the ellipse of a test particle [6-8], has been the subject of intense activity, both theoretically and observationally, in recent times [9].

Here, we will consider as reference path a Newtonian hyperbolic trajectory. In order to work out the effects of the gravitomagnetic field on it, we will numerically integrate the equations of motion in Cartesian rectangular coordinates [10] for some particular orbital geometries over a time span including the epoch of closest approach to $M$ which is assumed located at the origin of the coordinate system.

2.1. Qualitative Features for Equatorial and Polar Osculating Orbits. First, we will consider a trajectory lying in the 


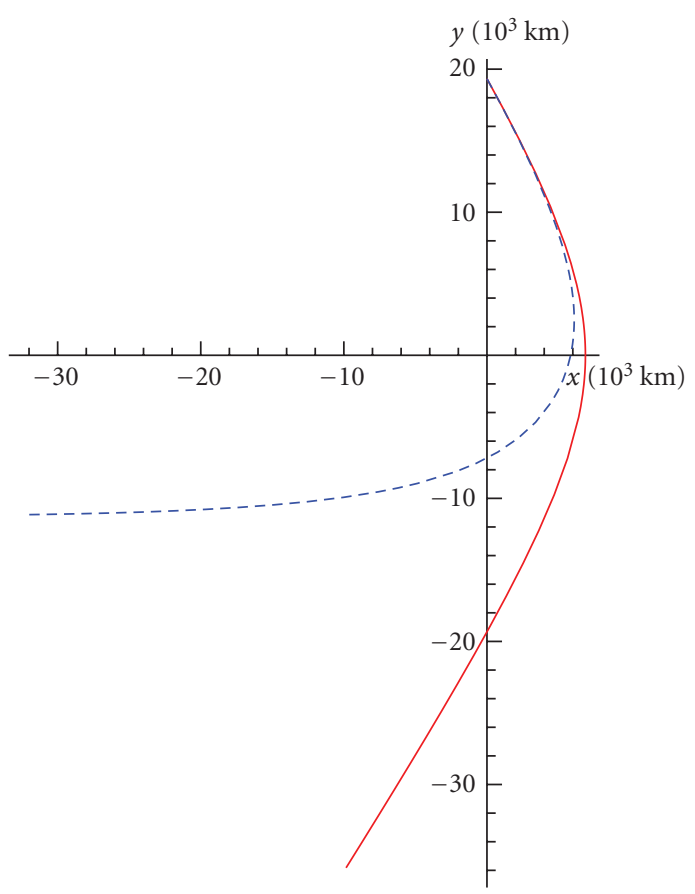

FIGURE 3: Effect of the gravitomagnetic force on the hyperbolic motion of a test particle around an astronomical rotating body located at the origin of the depicted frame. The body spin $\mathbf{S}$ is directed along the positive $z$ axis, that is, outside the figure. Red line: unperturbed hyperbola. Blue dashed line: perturbed orbit. For illustrative purposes, we choose the Earth as central body and rescaled the magnitude of its gravitomagnetic force by $10^{10}$ so that $A^{\mathrm{GM}} / A^{\mathrm{N}}=0.4$ at perigee. We adopted the initial conditions $x_{0}=0, y_{0}=p=a\left(e^{2}-1\right), z_{0}=0, v_{x 0}>0, v_{y 0}<0, v_{z 0}=0$; the particle moves in the equatorial plane of the spinning Earth in such a way that the radius vector rotates in the opposite sense with respect to the Earth, that is, clockwise. We used $a=8,493.326 \mathrm{~km}$, $e=1.81$. The perturbed orbit is deflected inward with respect to the unperturbed one.

equatorial plane of the rotating body for the cases of corotation (Figure 2) and counterrotation (Figure 3) of the particle radius vector $\mathbf{r}$ with respect to the diurnal rotation of $M$ whose spin $\mathbf{S}$ is assumed to be directed along the positive $z$ axis (anticlockwise diurnal rotation). Just for illustrative purposes, we assumed the Earth as source of the gravitational field and rescaled its gravitomagnetic force by a factor $10^{10}$ in such a way to still keeping the condition $A^{\mathrm{GM}} / A^{\mathrm{N}}<1$ valid over the entire orbit. It turns out that the perturbed trajectory remains confined in the equatorial plane of the central body; for corotation, the path is deflected outward with respect to the unperturbed hyperbola, while for counterrotation, it is deflected inward. Indeed, for equatorial orbits, $\mathbf{A}^{\mathrm{GM}}$ is entirely in-plane because $\mathbf{B}_{\mathrm{g}}$ is directed along the negative $z$ axis; for corotating particles, it is radially directed outward at the point of closest approach and decreases the gravitational pull felt by the orbiter, while for counterrotating probes, it is radially directed inward at the pericenter and increases the net gravitational acceleration. The flyby epoch is left almost unaffected. By

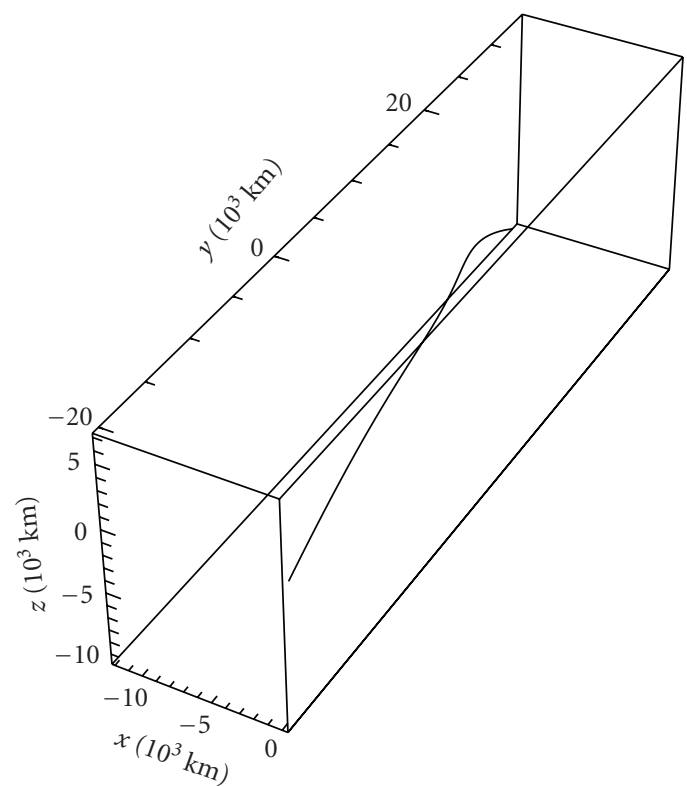

FIGURE 4: Effect of the gravitomagnetic force on the hyperbolic motion of a test particle around an astronomical rotating body located at the origin of the depicted frame. The body spin $S$ is directed along the positive $z$ axis, that is, outside the figure. For illustrative purposes, we choose the Earth as central body and rescaled the magnitude of its gravitomagnetic force by $10^{10}$ so that $A^{\mathrm{GM}} / A^{\mathrm{N}}=0.4$ at perigee. We adopted the initial conditions $x_{0}=$ $0, y_{0}=-p=-a\left(e^{2}-1\right), z_{0}=0, v_{x 0}=0, v_{y 0}>0, v_{z 0}>0$ to have the spacecraft initially moving in the osculating $\{y z\}$ plane. The perturbed trajectory is displaced along the $x$ axis on the upper left corner.

taking the difference between the integrated perturbed and unperturbed orbits sharing the same initial conditions, it can be shown that in the corotating case the radial velocity, which is one of the direct observables in real planet-spacecraft close encounters, experiences an increase with respect to the unperturbed one just around the flyby epoch, while the radial components of $v_{\infty i}$ and $v_{\infty}$ are left unaffected. In the counterrotating case $v_{r}$ decreases at the closest approach. Concerning the body-centric range $r$, it turns out that it suddenly increases (decreases) around the flyby epoch for the co-(counter-)rotating case and remains about at that level also after the flyby.

Let us, now, consider the case in which the unperturbed hyperbola entirely lies in an azimuthal plane, for example, the $\{y z\}$ plane, containing the spin $\mathbf{S}$ of the central body. Now, since $\mathbf{v}$ is contained in the same plane of $\mathbf{B}_{\mathrm{g}}$, the gravitomagnetic acceleration is out-of-plane, so that it can be expected that the perturbed trajectory will be displaced along the $x$ axis. This fact is confirmed by a numerical integration shown in Figures 4 and 5 from which the displacement of the orbit perpendicularly to the initial osculating plane is apparent.

2.2. The Flyby Anomaly: the NEAR Case. Many spacecrafts launched in interplanetary missions make use of one or more 


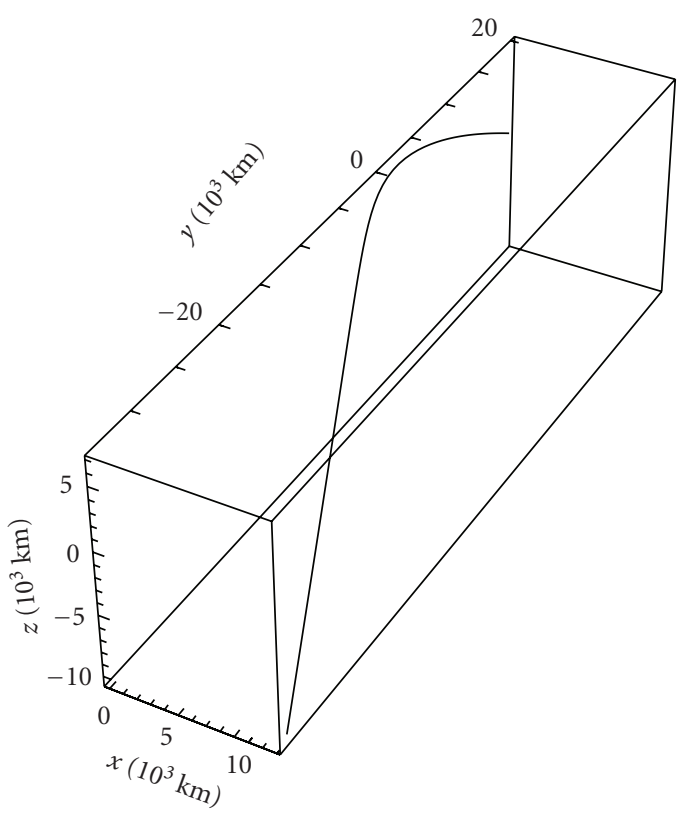

FIGURE 5: Effect of the gravitomagnetic force on the hyperbolic motion of a test particle around an astronomical rotating body located at the origin of the frame shown with its spin directed along the positive $z$ axis, that is, outside the figure. For illustrative purposes, we choose the Earth as central body and rescaled the magnitude of its gravitomagnetic force by $10^{10}$ so that $A^{\mathrm{GM}} / A^{\mathrm{N}}=$ 0.4 at perigee. We adopted the initial conditions $x_{0}=0, y_{0}=$ $p=a\left(e^{2}-1\right), z_{0}=0, v_{x 0}=0, v_{y 0}<0, v_{z 0}>0$ to have the spacecraft initially moving in the osculating $\{y z\}$ plane. The perturbed trajectory is displaced along the $x$ axis on the lower right corner.

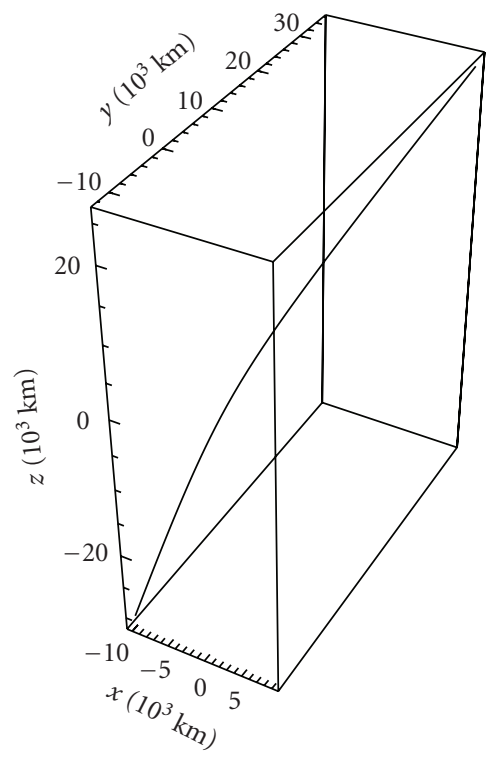

FIGURE 6: Unperturbed hyperbola of NEAR; its osculating plane is tilted by $108 \mathrm{deg}$ to the $\{x, y\}$ plane assumed coincident with the Earth equator. The starting point is in the right upper corner $\left(x_{0}>\right.$ $\left.0, y_{0}>0, z_{0}>0\right)$. The Earth has to be imagined located at the origin of the coordinate system with its spin $S$ directed along the positive $z$ axis.

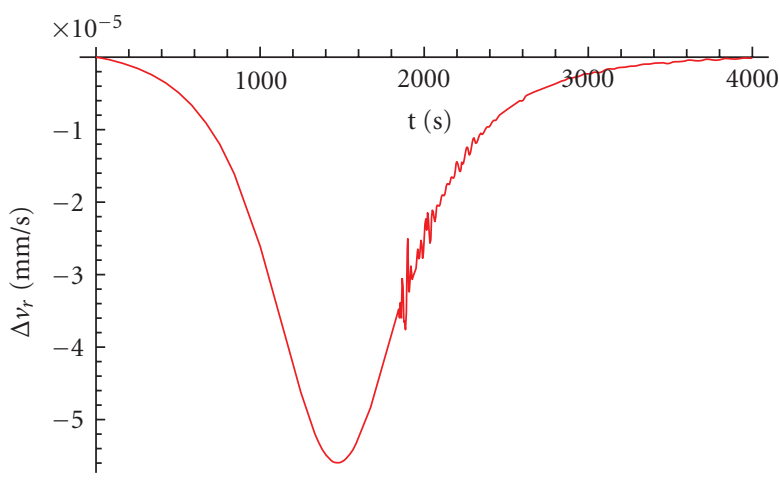

Figure 7: Change in the radial velocity $\Delta v_{r}$ induced by the gravitomagnetic force on NEAR at the Earth flyby (1998-Jan-23 07:22:56 coordinate time (CT)). It has been obtained by taking the difference between the integrated perturbed and unperturbed trajectories sharing the same initial conditions of Table 1. They have been obtained with the HORIZONS software by NASA JPL at 1998-Jan-23 07:00:00 CT and correspond to an instant 1353 seconds before the flyby. Reference frame: ICRF/J2000.0. Coordinate system: Earth Mean Equator and Equinox of Reference Epoch. The maximum effect $\Delta v_{r}^{\max }=-5 \times 10^{-5} \mathrm{~mm} \mathrm{sec}^{-1}$ occurred just at the flyby.

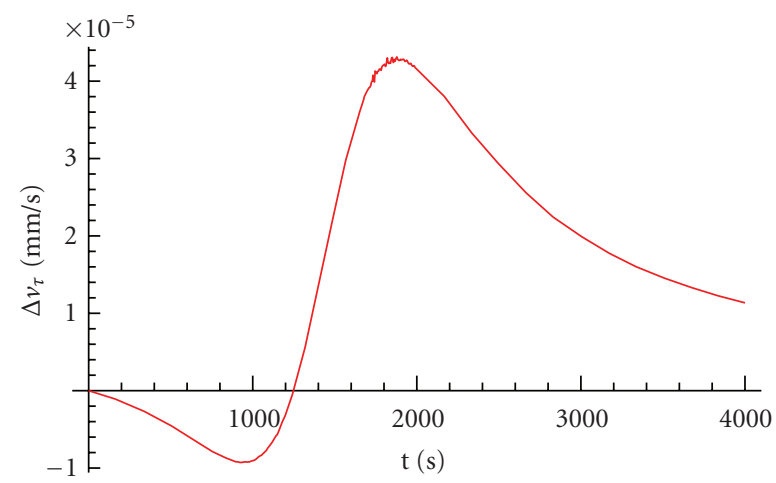

FIGURE 8: Change in the transverse velocity $\Delta v_{\tau}$ induced by the gravitomagnetic force on NEAR at the Earth flyby (1998-Jan-23 07:22:56 CT). The maximum value reached is of the order of $10^{-5} \mathrm{~mm} \mathrm{sec}^{-1}$.

Earth flybys in order to gain or lose the heliocentric energy required to reach their far targets (planets, asteroids, comets) without using huge and expensive amounts of propellant $[11,12]$. In the case of GALILEO (twice), NEAR, Cassini and MESSENGER unexplained variations in $v_{\infty}$ were detected $[2,3]$; the largest one was measured at the close encounter of NEAR with the Earth that occurred in 1998 and amounts to

$$
\Delta v_{\infty}=13.46 \pm 0.01 \mathrm{~mm} \mathrm{sec}^{-1} .
$$

The unperturbed hyperbola of NEAR is depicted in Figure 6. 


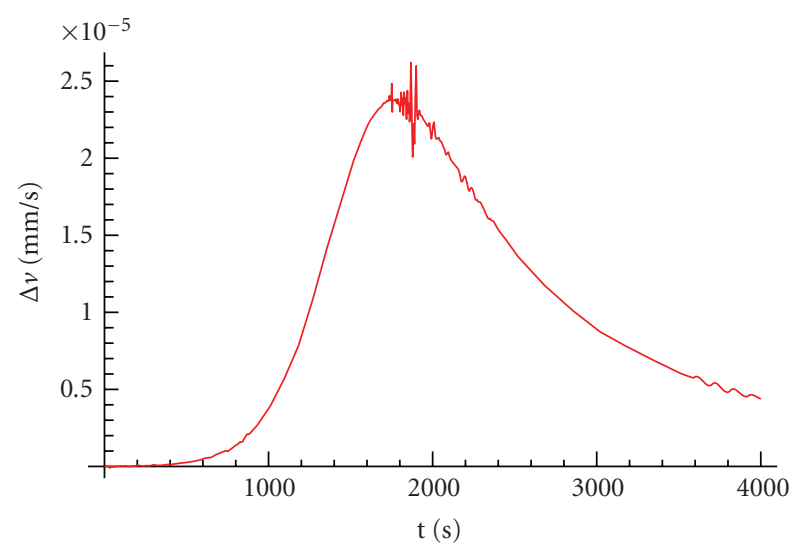

FIgURe 9: Change in the speed $\Delta v$ induced by the gravitomagnetic force on NEAR at the Earth flyby (1998-Jan-23 07:22:56 CT). The maximum effect $\Delta v^{\max }=2 \times 10^{-5} \mathrm{~mm} \mathrm{sec}^{-1}$ occurred just at the flyby while $v_{\infty}$ is left almost unchanged.

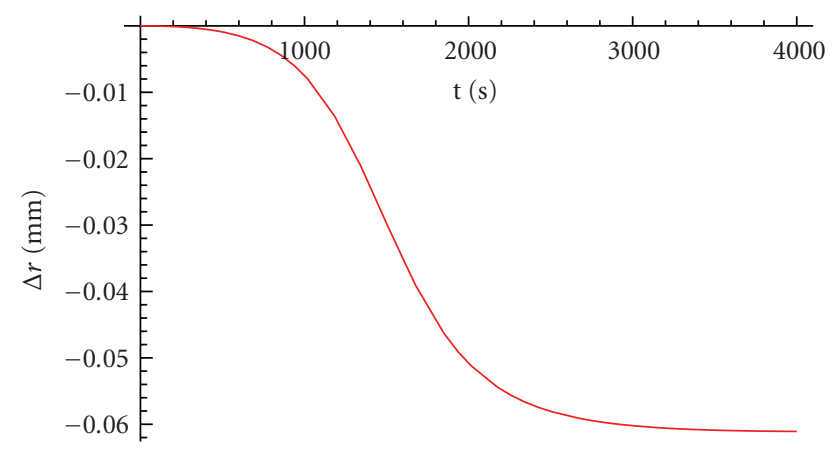

Figure 10: Change in the radial distance $\Delta r$ induced by the gravitomagnetic force on NEAR at the Earth flyby (1998-Jan-23 07:22:56 CT). The maximum effect $\Delta r^{\max }=-6 \times 10^{-2} \mathrm{~mm}$ took place after the flyby.

Anderson et al. [3] derived an empirical formula which satisfactorily fit all the six flyby anomalies measured so far. It is

$$
\begin{aligned}
\frac{\Delta v_{\infty}}{v_{\infty}} & =\left(\frac{2 \omega R}{c}\right)\left(\cos \delta_{\mathrm{i}}-\cos \delta_{\mathrm{o}}\right) \\
& =\left(3.099 \times 10^{-6}\right)\left(\cos \delta_{\mathrm{i}}-\cos \delta_{\mathrm{o}}\right),
\end{aligned}
$$

where $\omega$ and $R$ are the Earth angular speed and equatorial radius, respectively, while $\delta_{\mathrm{i}}$ and $\delta_{\mathrm{o}}$ are the ingoing and ongoing geocentric declinations, respectively. Concerning possible explanations in terms of known physics, many dynamical effects (tides, atmospheric drag, Earth albedo, solar wind, terrestrial magnetic field, spin-rotation coupling between electromagnetic waves and spacecraft, and Earth rotations) were preliminarily considered by Lämmerzahl et al. [13]; an order-of-magnitude approach was followed by confronting the magnitude of the accelerations induced by standard forces considered with the one which is assumed to be responsible of the flyby anomaly, that is, $\approx 10^{-4} \mathrm{~m} \mathrm{sec}^{-2}$. As a consequence, all the effects investigated were discarded. However, we note that detailed analyses are in order. Indeed,

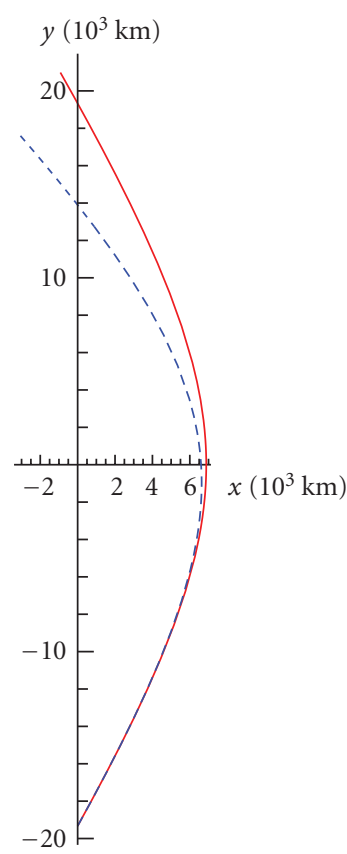

FIGURE 11: Effect of the gravitoelectric force on the hyperbolic motion of a test particle around an astronomical body located at the origin of the frame shown. Red line: unperturbed hyperbola. Blue dashed line: perturbed orbit. For illustrative purposes, we choose the Earth as central body and rescaled the magnitude of its gravitoelectric force by $10^{8}$ so that $A^{\mathrm{GE}} / A^{\mathrm{N}}=0.1$ at perigee. We adopted the initial conditions $x_{0}=0, y_{0}=-p=-a\left(e^{2}-\right.$ $1), z_{0}=0, v_{x 0}>0, v_{y 0}>0, v_{z 0}=0$. We used $a=8,493.326 \mathrm{~km}$, $e=1.81$. The perturbed orbit is deflected inward with respect to the unperturbed one.

even if some dynamical effect, standard or not, was found to produce an acceleration with the right order of magnitude, it might happen that its signature on the observable quantities is not correct; that is, it may induce, for instance, a decrease of the radial velocity. Mbelek [14] suggested that the special relativistic Doppler effect may explain the formula by Anderson et al. [3]. Among various explanations in terms of nonconventional physics put forth so far, McCulloch [15] proposed a mechanism based on the hypothesis that inertia is due to a form of Unruh radiation and varies with acceleration due to a Hubble-scale Casimir effect. It qualitatively reproduces the latitude-dependence of (9) and is quantitatively in agreement with three of the six measured flybys.

Since (9) contains a term including quantities like the speed of light and the first power of the Earth angular speed which enters general relativistic gravitomagnetic effects, it seems interesting to apply our previous results concerning the influence of $\mathbf{B}_{\mathrm{g}}$ on hyperbolic orbits to the NEAR flyby. Note that the gravitomagnetic force was not modeled in the software used to process the NEAR data. By using the state vector of NEAR at the flyby epoch (J D Anderson, private communication to the author, November 2008) referred to a geocentric equatorial frame $\{x, y, z\}$, and (7), it turns out 
TABLE 1: Initial conditions used for NEAR obtained with the HORIZONS software by NASA, JPL at 1998-Jan-23 07:00:00 coordinate time (CT, defined as the uniform time scale and independent variable of the ephemerides http://ssd.jpl.nasa.gov/?horizons_doc\#timesys), that is, 1353 seconds before the flyby. Reference frame: ICRF/J2000.0. Coordinate system: Earth Mean Equator and Equinox of Reference Epoch.

\begin{tabular}{lccccc}
\hline$x_{0}(\mathrm{~km})$ & $y_{0}(\mathrm{~km})$ & $z_{0}(\mathrm{~km})$ & $v_{x 0}\left(\mathrm{~km} \mathrm{sec}^{-1}\right)$ & $v_{y 0}\left(\mathrm{~km} \mathrm{sec}^{-1}\right)$ & $v_{z 0}\left(\mathrm{~km} \mathrm{sec}^{-1}\right)$ \\
\hline $4,496.885594909381$ & $6,930.477153733549$ & $13,199.11503591246$ & -1.712684317202157 & -8.679677119077454 & -4.455285829060190 \\
\hline
\end{tabular}

that at the point of closest approach to Earth along the flyby trajectory

$$
\begin{aligned}
& A_{x}^{\mathrm{GM}}=3.3 \times 10^{-10} \mathrm{~m} \mathrm{sec}^{-2}, \\
& A_{y}^{\mathrm{GM}}=7.5 \times 10^{-11} \mathrm{~m} \mathrm{sec}^{-2}, \\
& A_{z}^{\mathrm{GM}}=-1.7 \times 10^{-10} \mathrm{~m} \mathrm{sec}^{-2},
\end{aligned}
$$

so that

$$
A^{\mathrm{GM}}=3.8 \times 10^{-10} \mathrm{~m} \mathrm{sec}^{-2}
$$

We will now use a numerical integration of the equations of motion perturbed by (7). We look at a time span starting from the flyby epoch and extending in the future after it. The chosen initial conditions are in Table 1 . We will consider the changes of the velocity along the radial $\widehat{\mathbf{r}}$, transverse $\hat{\boldsymbol{\tau}}$, and out-of-plane $\hat{v}$ directions; $\hat{v}$ is directed along the orbital angular momentum, while $\hat{\boldsymbol{\tau}}=\hat{\boldsymbol{v}} \times \hat{\mathbf{r}}$ is not directed, in general, along $\mathbf{v}$.

The results for $\Delta v_{r}, \Delta v_{\tau}, \Delta v$, and $\Delta r$ are shown in Figures $7,8,9$, and 10 , respectively. They have been obtained by subtracting the unperturbed orbit from the perturbed one, both numerically integrated with the initial conditions of Table 1.

The gravitomagnetic force of the Earth decreased the radial velocity of NEAR by $\approx 10^{-5} \mathrm{~mm} \mathrm{sec}^{-1}$ just at the flyby, while the transverse velocity was augmented after the flyby up to $10^{-5} \mathrm{~mm} \mathrm{sec}^{-1}$ level; it turns out that the normal velocity was affected at an even smaller level. The total speed $v$ was increased up to $10^{-5} \mathrm{~mm} \mathrm{sec}^{-1}$, that is, six orders of magnitude smaller than the observed increment. The geocentric range of the spacecraft was reduced by about $10^{-2} \mathrm{~mm}$.

\section{The Gravitoelectric Force}

In the post-Newtonian approximation of order $\mathcal{O}\left(c^{-2}\right)$, the acceleration induced by the gravitoelectric component of the field of a static mass $M$ is, in standard isotropic coordinates, [10]

$$
\mathbf{A}^{\mathrm{GE}}=\frac{G M}{c^{2}}\left[\frac{4 G M}{r^{4}} \mathbf{r}-\frac{v^{2}}{r^{3}} \mathbf{r}+\frac{4(\mathbf{r} \cdot \mathbf{v})}{r^{3}} \mathbf{v}\right]
$$

Concerning the influence of (12) on the hyperbolic motion, it has only radial and transverse components, so that no departures from the osculating plane occur. From Figure 11, it turns out that the test particle is deflected inward with respect to the unperturbed hyperbola.

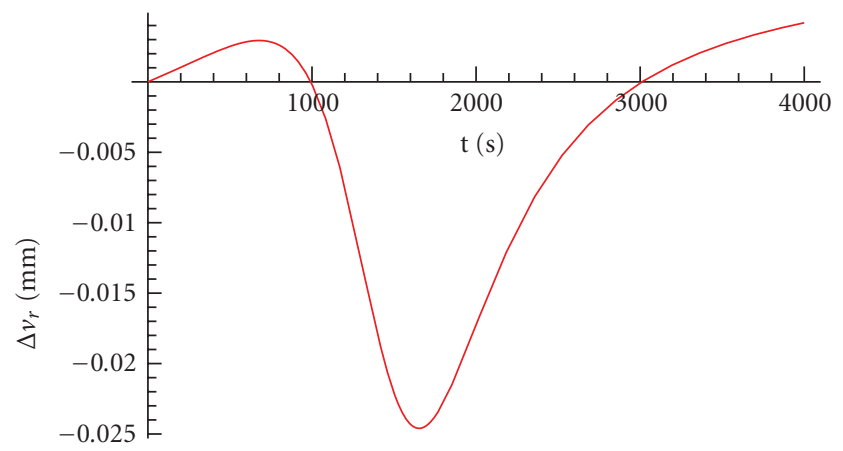

FIgure 12: Change in the radial velocity $\Delta v_{r}$ induced by the gravitoelectric force on NEAR at the Earth flyby (1998-Jan-23 07:22:56 CT). It is the difference between the integrated perturbed and unperturbed trajectories sharing the same initial conditions of Table 1 . They have been obtained with the HORIZONS software by NASA JPL at 1998-Jan-23 07:00:00 CT and correspond to an instant 1353 seconds before the flyby. Reference frame: ICRF/J2000.0. Coordinate system: Earth Mean Equator and Equinox of Reference Epoch.

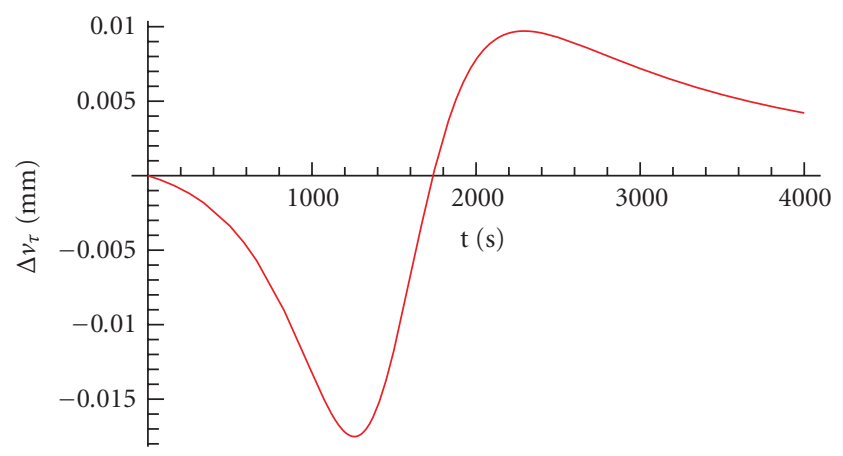

FIGURE 13: Change in the transverse velocity $\Delta v_{\tau}$ induced by the gravitoelectric force on NEAR at the Earth flyby (1998-Jan-23 07:22:56 CT).

The gravitoelectric acceleration experienced by NEAR at the point of closest approach to earth along the flyby trajectory was

$$
\begin{aligned}
& A_{x}^{\mathrm{GE}}=9.5 \times 10^{-10} \mathrm{~m} \mathrm{sec}^{-2}, \\
& A_{y}^{\mathrm{GE}}=-5.26 \times 10^{-9} \mathrm{~m} \mathrm{sec}^{-2}, \\
& A_{z}^{\mathrm{GE}}=3.42 \times 10^{-9} \mathrm{~m} \mathrm{sec}^{-2},
\end{aligned}
$$

so that

$$
A^{\mathrm{GE}}=6.35 \times 10^{-9} \mathrm{~m} \mathrm{sec}^{-2} .
$$

The impact of the gravitoelectric force on $v_{r}, v_{\tau}, v$, and $r$ of NEAR at its flyby are depicted in Figures 12, 13, 14, 


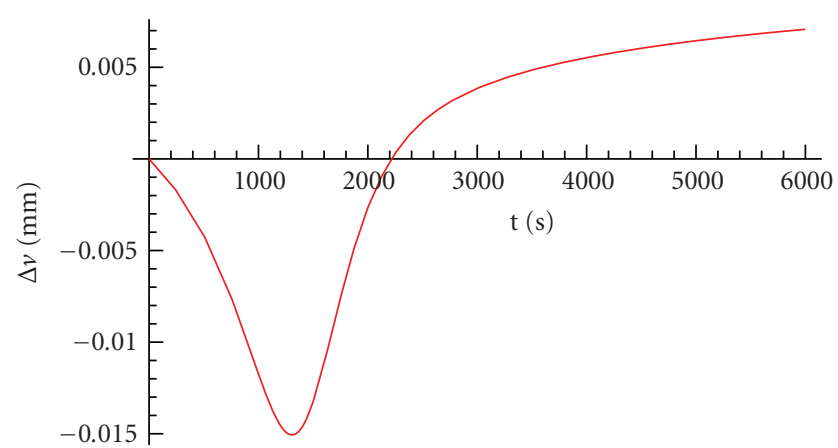

Figure 14: Change in the speed $\Delta v$ induced by the gravitoelectric force on NEAR at the Earth flyby (1998-Jan-23 07:22:56 CT).

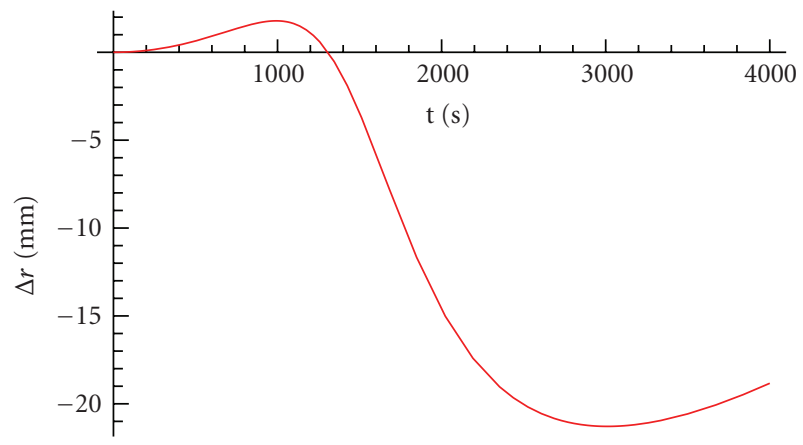

Figure 15: Change in the radial distance $\Delta r$ induced by the gravitoelectric force on NEAR at the Earth flyby (1998-Jan-23 07:22:56 CT).

and 15, respectively. Their patterns are quite different from the gravitomagnetic ones; the outgoing asymptotic velocity is changed by an amount of the order of $10^{-3} \mathrm{~mm} \mathrm{sec}^{-1}$. (Contrary to the gravitomagnetic one, the gravitoelectric force was modeled in the software used for processing the data.) The maximum variations of the range rate, the transverse velocity, the speed, and the range are of the order of $10^{-2} \mathrm{~mm} \mathrm{sec}^{-1}$ and $10 \mathrm{~mm}$, respectively.

\section{Discussions and Conclusions}

We investigated qualitatively and quantitatively the impact of general relativity, in its weak-field and slow-motion approximation, on unbound hyperbolic orbits around a massive spinning body. We considered both the gravitomagnetic and the gravitoelectric terms; the first one is responsible of the Lense-Thirring precessions of elliptic orbits, while the second one causes the well-known Einstein precession of the perihelion of Mercury of $43.98 \operatorname{arcsec} c t y^{-1}$. The gravitomagnetic force deflects an equatorial trajectory inward or outward with respect to the unperturbed hyperbola according to the mutual orientation of the orbital angular momentum $\mathbf{L}$ of the particle with respect to the spin $\mathbf{S}$ of the central body. For osculating orbits lying in a plane which contains $\mathbf{S}$, there is also a displacement in the out-of-plane direction. The gravitoelectric force is not sensitive to the $\mathbf{L}-\mathbf{S}$ orientation and deflects the trajectory inward with respect to the unperturbed hyperbola.

We applied our results to the flyby anomaly experienced by the NEAR spacecraft at its close encounter with the Earth on January 1998 when its asymptotic outgoing velocity $v_{\infty}$ o was found larger than the ingoing one by $13.46 \pm$ $0.01 \mathrm{~mm} \mathrm{sec}^{-1}$; contrary to the gravitoelectric force, the gravitomagnetic one was not modeled in the software used to process the NEAR data. From numerical integrations of the perturbed equations of motion in a geocentric equatorial frame with rectangular Cartesian coordinates over a time span extending in the future after the flyby epoch, we quantitatively investigated the changes in the radial and transverse components of the velocities $v_{r}$ and $v_{\tau}$, the speed $v$, and the range $r$ of NEAR induced by the general relativistic gravitoelectromagnetic forces. Concerning the range, its variations are at the $10^{-2}$ level for the gravitomagnetic force and $10^{1} \mathrm{~mm}$ level for the gravitoelectric one. The radial and transverse velocities and the speed are affected at $10^{-2}$ (gravitoelectric) to $10^{-5}$ (gravitomagnetic) $\mathrm{mm} \mathrm{sec}^{-1}$ levels.

\section{References}

[1] B. Mashhoon, "Gravitoelectromagnetism: a brief review," in The Measurement of Gravitomagnetism: A Challenging Enterprise, L. Iorio, Ed., pp. 29-39, NOVA, Hauppauge, NY, USA, 2007.

[2] J. D. Anderson, J. K. Campbell, and M. M. Nieto, "The energy transfer process in planetary flybys," New Astronomy, vol. 12, no. 5, pp. 383-397, 2007.

[3] J. D. Anderson, J. K. Campbell, J. E. Ekelund, J. Ellis, and J. F. Jordan, "Anomalous orbital-energy changes observed during spacecraft flybys of earth," Physical Review Letters, vol. 100, no. 9, Article ID 091102, 4 pages, 2008.

[4] A. E. Roy, Orbital Motion, Institute of Physics, Bristol, UK, 4th edition, 2005.

[5] H. I. M. Lichtenegger and L. Iorio, "Post-Newtonian orbital perturbations," in The Measurement of Gravitomagnetism: A Challenging Enterprise, L. Iorio, Ed., pp. 87-100, NOVA, Hauppauge, NY, USA, 2007.

[6] J. Lense and H. Thirring, "Über den Einfluss der Eigenrotation der Zentralkörper auf die Bewegung der Planeten und Monde nach der Einsteinschen Gravitationstheorie," Physikalische Zeitschrift, vol. 19, pp. 156-163, 1918.

[7] B. Mashhoon, F. W. Hehl, and D. S. Theiss, "On the gravitational effects of rotating masses: the Thirring-Lense papers," General Relativity and Gravitation, vol. 16, no. 8, pp. 711-750, 1984.

[8] B. Mashhoon, F. W. Hehl, and D. S. Theiss, "On the gravitational effects of rotating masses: the Thirring-Lense papers," in Nonlinear Gravitodynamics, R. J. Ruffini and C. Sigismondi, Eds., pp. 349-388, World Scientific, Singapore, 2003.

[9] L. Iorio, Ed., The Measurement of Gravitomagnetism: A Challenging Enterprise, NOVA, Hauppauge, NY, USA, 2007.

[10] M. Soffel, Relativity in Astrometry, Celestial Mechanics and Geodesy, Springer, Berlin, Germany, 1989.

[11] G. A. Flandro, "Fast reconnaissance missions to the outer solar system utilizing energy derived from the gravitational field of Jupiter," Astronautica Acta, vol. 12, no. 4, pp. 329-337, 1966. 
[12] J. A. van Allen, "Gravitational assist in celestial mechanics-a tutorial," American Journal of Physics, vol. 71, no. 5, pp. 448$451,2003$.

[13] C. Lämmerzahl, O. Preuss, and H. Dittus, "Is the physics within the solar system really understood?" in Lasers, Clocks and Drag Free Control: Exploration of Relativistic Gravity in Space, H. Dittus, C. Lämmerzahl, and S. G. Turyshev, Eds., pp. 75-104, Springer, Berlin, Germany, 2008.

[14] J. P. Mbelek, "Special relativity may account for the spacecraft flyby anomalies," http://arxiv.org/abs/0809.1888.

[15] M. E. McCulloch, "Modelling the flyby anomalies using a modification of inertia," Monthly Notices of the Royal Astronomical Society: Letters, vol. 389, no. 1, pp. L57-L60, 2008. 